\title{
Play in Rats: Association across Contexts and Types, and Analysis of Structure
}

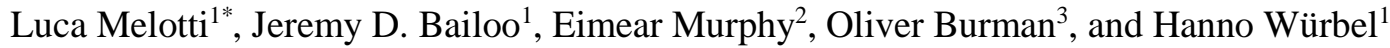 \\ ${ }^{1}$ University of Bern \\ ${ }^{2}$ Utrecht University \\ ${ }^{3}$ University of Lincoln \\ *Corresponding author (Email: luca.melotti@vetsuisse.unibe.ch)
}

Citation - Melotti, L., Bailoo, J. D., Murphy, E., Burman, O., \& Würbel, H. (2014). Play in rats: Association across contexts and types, and analysis of structure. Animal Behavior and Cognition, 1(4), 489-501. doi: 10.12966/abc.11.06.2014

\begin{abstract}
Play has been proposed as a promising indicator of positive animal welfare. We aimed to study play in rats across contexts (conspecific/heterospecific) and types (social: pinning, being pinned; solitary: scampering), and we investigated its structure using behavioral sequence analysis. Group-housed (three per cage) adolescent male Lister Hooded rats $(n=21)$ were subjected to a Play-In-Pairs test: after a 3 hour isolation period, a pair of cagemates was returned to the home cage and both social and solitary play were scored for 20 min. This procedure was repeated for each pair combination across three consecutive days, and individual play scores were calculated. Heterospecific play was measured using a Tickling test: rats were individually tickled by the experimenter through bouts of gentle, rapid finger movements on their underside, and the number of positive $50 \mathrm{kHz}$ frequency modulated vocalizations and experimenter-directed approach behaviors were recorded. Both of the above tests were compared with social play in the home cage. While conspecific play in both the Play-In-Pairs test and home cage were correlated, both seemed to be unrelated to heterospecific play in the Tickling test. During the Play-In-Pairs test, although both solitary and social play types occurred, they were unrelated, and solitary locomotor play of one rat did not predict the subsequent play behavior of its cage mate. Analysis of play structure revealed that social play occurred more often in bouts of repeated behaviors while solitary play sequences did not follow a specific pattern. If play is to be used as an indicator of positive welfare in rats, context, type and structure differences should be taken into account.
\end{abstract}

Keywords Play, Animal welfare, Rat, Social play, Tickling

Play in humans can be broadly defined as a range of intrinsically-motivated, not goal-directed actions that are both pleasurable and enjoyable, and in which an individual actively and voluntarily engages (Garvey, 1990). Similarly to humans, play has been found to occur in a large variety of animal species, mainly within mammals and birds (Held \& Spinka, 2011; Spinka, Newberry, \& Bekoff, 2001), and while specific play behaviors may differ, the properties of play are maintained and generalizable across species (Burghardt, 2005; Graham \& Burghardt, 2010). Play is autotelic (Burghardt, 2005), that is the performance of a playful action is rewarding in itself, and is extremely labile, as it disappears as soon as the animal is exposed to threats to its fitness (Burghardt, 2005; Klein, Padow, \& Romeo, 2010; Panksepp \& Burgdorf, 1999). Three main types of play behavior have been investigated in animals, namely social play, locomotor-rotational play and object-directed play (Held \& Spinka, 2011), the most commonly studied of which is social play (Burghardt, 2005). 
Although the properties of play may render its study rather challenging, its use as a potential indicator of good animal welfare is becoming increasingly relevant (Boissy et al., 2007; Fagen, 1981; Held \& Spinka, 2011; Lawrence, 1987; Oliveira, Rossi, Silva, Lau, \& Barreto, 2010). Indeed, play has been associated with terms such as 'fun' (Bekoff, 2001; Spinka et al., 2001; Vanderschuren, 2010), 'pleasure' (Vanderschuren, 2010) and 'joy' (Panksepp, 2005), which imply the subjective experience of positive emotions (Boissy et al., 2007; Burgdorf \& Panksepp, 2006; Fraser \& Duncan, 1998). Play can also act as a reinforcer in the same way as food and sex (Vanderschuren, 2010), since the same neurotransmitters that mediate the motivational and hedonic properties of reward have been shown to also affect social play in rats (Burgdorf \& Panksepp, 2006; Vanderschuren, 2010).

Such properties of play have been studied extensively in rats (Douglas, Varlinskaya, \& Spear, 2004; Knutson, Burgdorf, \& Panksepp, 1998; Panksepp, 1981; Pellis, Field, Smith, \& Pellis, 1997; Pellis \& McKenna, 1992; Pellis \& Pellis, 1987; Vanderschuren, 2010), and rats can serve as a promising model for the study of play and emotional state for a number of reasons. Firstly, like most social mammals, rats exhibit social play, commonly referred to as play-fighting, or rough-and-tumble play (Pellis \& Pellis, 2007), which is ideal for comparative research. Play fighting in rats has been thoroughly studied and characterized (Pellis \& McKenna, 1992; Pellis et al., 1997), and is distinguishable from aggressive behavior (Pellis \& Pellis, 1987). Locomotor-rotational play, though less studied, is also expressed by this species and consists of a number of 'run' and 'jerk-jump' behaviors (Pellis \& Pellis, 1983). Secondly, vocalizations during play in rats have been proposed to represent accurate and measurable indicators of acute emotional state (Burgdorf, Panksepp, \& Moskal, 2011; Knutson, Burgdorf, \& Panksepp, 2002). In a number of contexts, including play fighting and mating, rats emit two clearly distinguishable ultrasonic calls differing in frequency, namely $22 \mathrm{kHz}$ and $50 \mathrm{kHz}$ calls. $22 \mathrm{kHz}$ calls are emitted in the face of threat stimuli and during avoidance behavior, while $50 \mathrm{kHz}$ calls have been shown to be associated with high behavioral arousal and positive appetitive behavior (Knutson et al., 2002). In particular, frequency modulated $50 \mathrm{kHz}$ calls have been suggested to reflect positively valenced emotional states (Burgdorf et al., 2008; 2011). Rats emit these frequency modulated $50 \mathrm{kHz}$ calls in both conspecific and heterospecific play contexts ('tickling' administered by the experimenter; Panksepp \& Burgdorf, 1999), and some evidence suggests that they will self-administer the playback of such calls if given the possibility to do so (Burgdorf et al., 2008).

While the above findings indicate that both conspecific and heterospecific play have positive valence, individual consistency of play across these contexts has been scarcely investigated in rats. The intrinsic propensity to play (i.e., the personality trait 'playfulness') across contexts may shed light on whether these are part of the same play dimension. While studies have reported that the same positive vocalizations produced during play (and sex) are also emitted during tickling (Burgdorf et al., 2008), only two studies have investigated whether conspecific social play (Rough-and-Tumble play) is related to vocalizations induced by tickling (Panksepp \& Burgdorf, 1999; Webber et al., 2012). While Panksepp and Burgdorf (1999) found a positive association between initiations of social play and vocalizations during tickling at 44 days old, this was not found at 58 days old, and little or no evidence was found for associations with other play behaviors (i.e., 'pinning'). Webber et al. (2012) found that while rats selectively bred for higher vocalization rates during tickling showed more initiations of play, compared with rats selected for lower vocalization rates, they showed lower durations of 'pinning' behavior during play. Further investigation is needed to clarify the relationship between play in conspecific and heterospecific contexts.

A period of social isolation is often used to induce high rates of play behavior in rats within a short time interval (Niesink \& van Ree, 1989; Panksepp \& Beatty, 1980; Vanderschuren, Niesink, Spruijt, 1995; Vanderschuren, Stein, Wiegant, \& van Ree, 1995). Though individual play motivation can be influenced by several factors, social isolation increases the motivation to initiate play in adolescent rats, and the increase in play depends on the duration of isolation (Niesink \& van Ree, 1989; Vanderschuren, Stein et al., 1995). Social isolation also increases the probability that play initiations result in the play partner being pinned, as opposed to adopting other defensive strategies (Pellis et al., 1997). However, increasing the motivation to play through social isolation might differ from the intrinsic propensity of a 
playful individual, and might even induce artefacts. Therefore it remains unclear whether animals that play more after being isolated also play more in standard home cage social context.

Playful contexts produce different types of play (e.g., solitary and/or social play) which are characterized by repeated yet non-stereotypic sequences of behavior (Burghardt, 2005). Play partners can influence each other in a contagious way, i.e., a highly playful rat may induce more frequent play in its partner (Pellis \& McKenna, 1992), therefore the analysis of sequences of play behavior between rats can shed light on possible associations between different types of play, namely social and locomotor play. Solitary locomotor play (described as 'running' by Pellis \& Pellis, 1983) is part of the locomotorrotational behavioral repertoire that can occur before and after conspecific social contact (Pellis \& Pellis, 1983; Wilson \& Kleiman, 1974). Little information is available on whether locomotor play events are associated with social play events (i.e., pinning and being pinned) in conspecific play. Moreover, behavioral sequence analysis within individuals can provide additional insight on the structure of a play bout, and how this structure may vary according to play type (i.e., solitary and social).

The aims of this study were (i) to assess whether individual play levels in conspecific and heterospecific contexts are related in rats, (ii) to search for associations between social and solitary play types, and (iii) to investigate play bout structure using behavioral sequence analysis.

\section{Method}

The experimental design and methods were approved by the responsible authorities of the Canton of Bern, Switzerland (License no. BE 63/12). Play behavior was measured in: (1) a conspecific test, 'Play-In-Pairs' test; (2) a heterospecific test, 'Tickling' test; and (3) the home cage (first hour of dark phase). Measures of anxiety-related behavior were derived from a 'Novelty-Induced Suppression-ofFeeding' test (Bodnoff, Suranyi-Cadotte, Quirion, \& Meaney, 1989).

We present our tests in order of relevance to the study. For a chronological overview, a graphical illustration of the experimental timeline is provided in Figure 1, indicating the order in which tests were performed and the ages of the animals at each test.

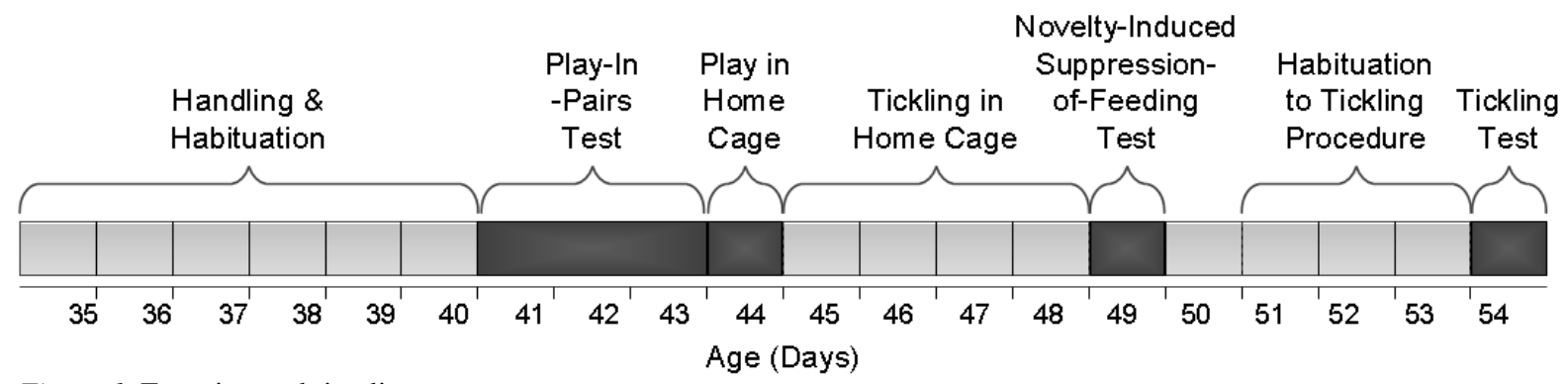

Figure 1. Experimental timeline.

\section{Animals and Housing}

Subjects were 21 male Lister Hooded rats born from seven litters at Charles River Laboratories, Sulzfeld, Germany. Males were used instead of females as males show higher levels of rough-and-tumble play during adolescence (Pellis et al., 1997). At weaning ( $24 \pm 1$ days; hereafter ' $d$ ') the animals were transported to the Division of Animal Welfare, University of Bern, Switzerland, and housed in seven standard type IV cages $(1 \mathrm{x} \mathrm{b} \times \mathrm{h}: 59.5 \mathrm{~cm} \times 38 \mathrm{~cm} \times 20 \mathrm{~cm}$, Tecniplast, Italy) in groups of three littermates, in a 12:12 light:dark cycle (lights off at $10 \mathrm{AM}$ ). Cage bedding consisted of wood shavings, and three paper tissues per cage were provided weekly, when cages were changed. Animals had ad libitum access to standard rodent food (KLIBA NAFAG, Switzerland) and tap water, and the housing room temperature was maintained at $22-24^{\circ} \mathrm{C}$. All animals were acclimatized to the experimenter and tested between five and eight weeks of age, during the dark light phase. The base, middle or tip of the rats' tail was marked weekly with a black marker pen for individual recognition of cage mates. 


\section{Conspecific 'Play-In-Pairs' Test}

Following six days of handling by the experimenter ( 15 min per cage, each rat gently picked up and handled), the last three of which included habituation to the noise of the video recording equipment, animals were subjected to a Play-In-Pairs Test over three consecutive days, between d $41-43$ of age. Repeated play sessions using different pair combinations of cage mates provides a measure of individual playfulness that is at least partly independent from the playfulness of the play partners (Pellis \& McKenna, 1992).

On the day before testing, animals were habituated to isolation cages (standard transparent type II cages, Tecniplast, Italy) with wood-shavings for bedding. Each animal was placed in a cage with one sweetened cereal for six minutes. On each test day, all animals were simultaneously isolated for $3 \mathrm{hr}$ in the isolation cages within the same housing room. This isolation period was chosen because it was shown to induce a substantial increase of social play in rats, without reaching a ceiling effect (Niesink \& van Ree, 1989; Vanderschuren, Niesink et al., 1995; Vanderschuren, Stein et al., 1995). After the isolation period, a pair of cage mates was returned to the home cage and both social and solitary play (see below) were scored for $20 \mathrm{~min}$. This procedure was repeated for each pair combination across the three test days, and scores from each combination were averaged to form individual play scores. Play behavior was video recorded using infrared cameras (VC, Germany) placed on one side of each home cage and supplemented by infrared lamps. Videos and behavior sequences were analyzed using the software Interact 9 (Mangold, Germany).

Social play scores were obtained by measuring the duration and frequency of the behavioral patterns Pinning and Being-Pinned. Pinning was defined as one rat standing over the exposed ventral area of the cage mate (Varlinskaya, Spear, \& Spear, 1999), with at least the two fore-paws placed on the chest of the pinned animal. Being-Pinned was defined as the complementary behavior, i.e., the pinned rat lying in supine position, with the cage mate standing/pressing on its ventral area.

Likely due to the relatively small space available in the home cage and to the high motivation to play induced by isolation, initiations of social play (i.e., attacks to the nape) almost exclusively led to pinning behavior, with very few instances of other playful defensive strategies such as partial rotation and evasion (preliminary experimenter's observations; see also Pellis \& Pellis, 1991; Pellis et al., 1997). For this reason, Pinning and Being-Pinned were considered to summarize social play in this experiment.

Solitary play scores were obtained by measuring the frequency of Scampering behavior. Scampering was defined as running rapidly alone, not towards the cage mate, and performing at least three hops. This behavior was not counted if triggered by an attack to the nape and/or chasing from the cage mate.

In order to assess whether play roles within an animal were equal (i.e., whether the durations of Pinning and Being Pinned for a given animal were similar), a Play-Role index was calculated using the following formula: $\left|\frac{p-b}{p+b}\right|$, where $p$ is Pinning duration and $b$ is Being Pinned duration for each animal. A higher Play-Role index indicates that an animal performs one role longer than the other, irrespective of which role (Pinning/Being Pinned) is performed the longest, while a lower index indicates greater similarity in duration of performance of both roles.

In order to understand the sequence of behaviors that may lead to or influence a bout of play behavior, two scores were calculated. First, frequencies of play behaviors (Pinning, Being Pinned, Scampering) of one animal, that followed Scampering behavior of its play mate, were counted and compared as proportions of the total frequency of play behaviors, and this was used to investigate associations in play behaviors between playmates. Second, intra-individual dyadic sequences of play behaviors were analyzed: for both social and solitary play, the frequencies of repetitions (e.g., Pinning event followed by another Pinning event, etc.) were counted and averaged to form a 'Repetition' score, and the same was done for the frequencies of alternations (e.g., Pinning event followed by Being Pinned, or Scampering events) to obtain an 'Alternation' score. These measures were used to investigate whether 
play consisted of bouts of the same behaviors repeated, or alternations between the different play behaviors.

\section{Heterospecific 'Tickling' Test}

Tickling Procedure. Animals were habituated to being tickled by the experimenter in their home cage for four days (20 min per cage on the first day, 6 min per cage on the following days). A tickling event consisted of gentle, rapid finger movements around the neck, followed by quickly turning the animal on its back and performing rapid finger movements on its underside for a few seconds (similar to Panksepp \& Burgdorf, 2000). Animals were then habituated to a tickling arena (all cage mates together) in a separate room for $20 \mathrm{~min}$. The tickling arena was a solid plastic box measuring $(1 \mathrm{x} \mathrm{w} \mathrm{x} \mathrm{h}) 49 \mathrm{~cm} \times 49$ $\mathrm{cm} \times 39 \mathrm{~cm}$. To speed up habituation to the arena, enrichment items were placed in the arena, namely two small solid plastic tunnels $(1=15.5 \mathrm{~cm}, \varnothing=9 \mathrm{~cm})$, one placed vertically with a small wooden ladder leading on top of it, and one placed horizontally, both of which were fixed to the floor. On the next day, animals were habituated to being tickled individually, by repeatedly placing them in the tickling arena for increasing periods of time (one, two, and three minute periods) and performing one or two bouts of tickling, depending on how comfortable the animal was (i.e., approaching/exploring experimenter's hand or avoiding/escaping from it). A bout of tickling was defined as performing three tickling events in rapid succession. Up to this training stage, while each animal was tickled in the tickling arena, cage mates were kept in their home cage beside the tickling arena.

On the day before testing (d 53), animals were habituated to be transported individually from the housing room to the test arena ( 1 min per animal), without the cage mates being present in the test room. Then, the following protocol was conducted: (a) the animal was placed in the test arena for $60 \mathrm{~s}$, with the hand of the experimenter in the arena; (b) a first tickling bout was performed; (c) immediately after, to check for approach behavior (see below), the experimenter moved the hand 3 times in a circle on the test arena's floor; (d) $45 \mathrm{~s}$ pause followed where the experimenter kept the hand still and gently touched the animals; (e) a second tickling bout was performed; (f) approach behavior was measured again in the same way as (c). The tickling session had a maximum duration of $2.5 \mathrm{~min}$.

The Tickling Test took place on d 54 and followed the same protocol as on the previous day (steps a, b, c, d, e, f). The number and type of vocalizations (see Section 2.3.2.) and number of fecal boli were recorded. Production of fecal boli was used as a proxy measure of stress/anxiety (Archer, 1973; Bodnoff et al., 1989). On d 53 and d 54, it was recorded whether an animal approached the experimenter's hand immediately after each tickling bout, and a combined Approach Behavior score (maximum 4 Approach Behaviors across 2 days) was calculated. In order to unambiguously measure approach behavior, the experimenter moved the hand in circles on the test arena's floor in the same way for all animals. This was done to better assess whether the first movement of the animal was intentionally directed towards the experimenter's hand.

Recording and Analysis of Vocalizations. Vocalizations were recorded using AvisoftUltraSoundGate $116 \mathrm{Hb}$ recorder with a high quality condenser microphone, and analyzed using AvisoftSASLab Pro software (Avisoft Bioacoustics, Germany). The microphone was placed over the centre of the tickling arena, pointing downwards and at a distance of $85 \mathrm{~cm}$ from the arena's floor. Recordings had frequency range of 5 to $90 \mathrm{kHz}$, sampling rate of $250 \mathrm{kHz}$ and a 16 bit resolution. Spectrograms were created with a fast Fourier transform (FFT)-length of 512 points and an overlap of 75\% (FlatTop window, $100 \%$ frame size), according to Wright, Gourdon, and Clarke (2010).

Vocalizations (positive or negative in valence) were manually counted from spectrograms. Vocalizations had to be at least $20 \mathrm{~ms}$ apart in order to be counted as separate vocalizations. Positive vocalizations were defined as frequency modulated $50 \mathrm{kHz}$ vocalizations containing a trill component. A trill consisted of at least two rapid frequency oscillations with a period of circa $5 \mathrm{~ms}$, either sinusoidal or appearing as repeated 'inverted-Us', as described in Wright et al. (2010). Positive vocalizations could contain steps, flat portions, ramps or jumps (Wright et al., 2010), yet they were scored as positive only if 
they contained a trill component. Negative vocalizations were defined as vocalizations with near-constant frequency ranging from 20 to $25 \mathrm{kHz}$ and lasting from 200 to $2000 \mathrm{~ms}$ (Poel \& Miczek, 1991; Wright et al., 2010).

The total number of vocalizations emitted during tickling bouts, and during the remaining time of the tickling session, was counted. As tickling bouts varied slightly in their duration (mean $\pm S E M$, here and thereafter: $17.7 \pm 0.4 \mathrm{~s}$ ), the data on vocalizations was analyzed and presented as number of vocalizations per second.

\section{Play in Home Cage}

On d 44, social play in the home cage was video recorded during the first hour of the dark phase, when general activity levels are relatively high (Hurst, Barnard, Hare, Wheeldon, \& West, 1996). The frequency of Pinning and Being-Pinned behaviors was scored for each rat according to the same definitions described in Section 2.2., and using the open source software Media Player Classic (mpchc.org). Total frequencies for the first $30 \mathrm{~min}$, and for the total $60 \mathrm{~min}$ duration, were calculated.

\section{Novelty-Induced Suppression-of-Feeding Test}

On d 49 a Novelty-Induced Suppression-of-Feeding test (Bodnoff et al., 1989) was performed to assess whether the above play measures were independent of anxiety level. The same arena which was subsequently used for the Tickling test (see Section 2.3.1.), without the enrichment objects, was also used for this test. Before the start of each test session, one sweetened cereal was placed on the centre of the arena's floor. Testing was performed under dimmed white light (1.4 lux at the centre of the arena) in a test room separate from the housing room.

Animals were individually transported from their home cage to the test room, and then placed in the test arena for three minutes. The number of fecal boli and the latency to eat the sweetened cereal were recorded and used as indicators of anxiety, with higher counts of fecal boli and higher latencies to eat corresponding to higher levels of anxiety (Archer, 1973; Bodnoff et al., 1989). Both the test arena and the transport cage were cleaned with $70 \%$ ethanol between test sessions to minimize any effect of odor communication between animals.

\section{Data Analysis}

All statistical analyses were carried out using SPSS (SPSS ${ }^{\circledR}$, version 22). Data were transformed (square root, logarithm or arcsine transformation) where they did not meet parametric assumptions. If transformations were not effective, data were analyzed using non-parametric tests. Frequencies of social play and solitary play were compared using a paired t-test.

Relationships between variables were explored using partial correlations which controlled for the nested factor, cage, or Spearman's rank correlations. For non-parametric data, a Kruskal-Wallis test was used to assess the effect of cage association. The factor 'cage' was not found to influence the relationship between any of our variables. The relationship between approach behavior and vocalizations during the Tickling Test was assessed by a Kruskal-Wallis test.

Play behaviors following Scampering of the play mate were compared by one-way repeated measures Analysis of Variance (ANOVA). For both social and solitary play, Repetition and Alternation scores were compared using paired t-tests.

\section{Results}

\section{Conspecific 'Play-In-Pairs' Test}

Social and Solitary Play. Social play (average between Pinning and Being Pinned frequencies) 
occurred more often than solitary play (Scampering frequency; $t(20)=7.55, p<0.001$ ). Frequencies and durations of social play were highly correlated $(r(18)=0.93, p<0.001)$, thus only durations (a more comprehensive measure), were used for further data analysis concerning social play.

Frequency of solitary play (Scampering behavior) was unrelated to social play duration $(r(18)=$ $0.16, n . s$.$) . The Play-Role index was positively correlated with Pinning duration (r(18)=0.45, p=0.04)$, but was unrelated to Being Pinned duration $(r(18)=-0.17$, n.s. $)$.

Analysis of Behavioral Sequences. The proportions of each play behavior (of total play events) that one animal performed following solitary play of its cage mate were not found to be significantly different $(F(2,40)=0.27$, n.s. $)$. Analysis of intra-individual dyadic sequences of behavior revealed that behavioral repetitions were more frequent than alternations for social play behaviors $(t(20)=9.89, p<$ 0.001 ; Figure 2a), but not for solitary play behavior $(t(20)=-0.18$, n.s.; Figure $2 b$ ).

a)

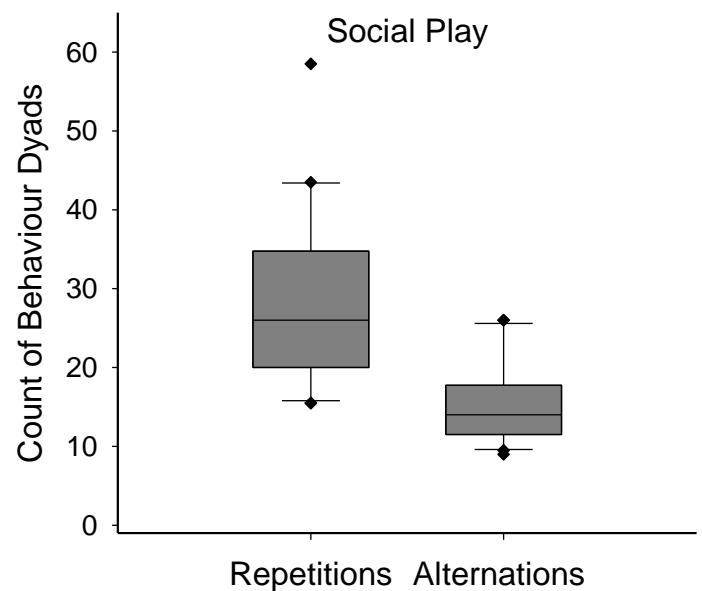

b)

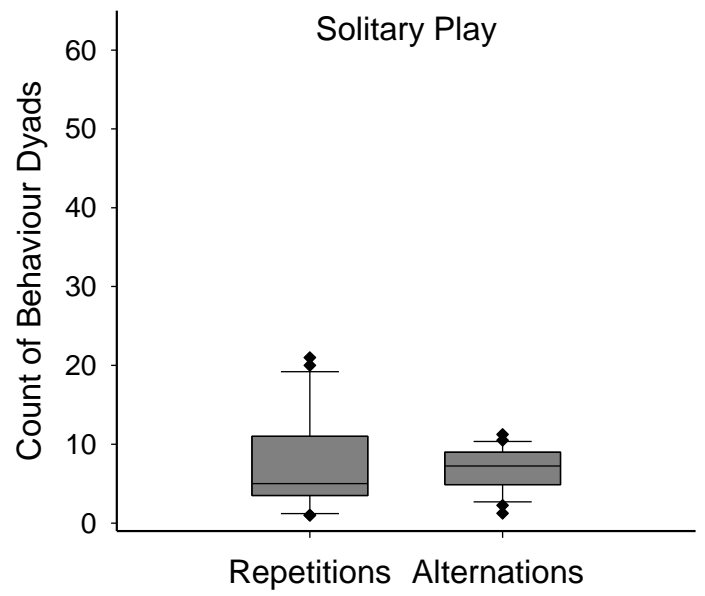

Figure 2. Frequencies of a) repetitions and alternations of social play behaviors (Pinning/Being Pinned) and b) repetitions of solitary play (Scampering) and alternations between solitary and social play behaviors.

\section{Heterospecific 'Tickling' Test}

Animals did not produce fecal boli or emit negative vocalizations during the Tickling Test. Positive vocalizations emitted during tickling bouts highly correlated with those emitted in the remaining time of the tickling session $\left(r_{s}=0.83, p<0.001\right)$. Therefore, only positive vocalizations emitted during 
tickling bouts were used for further analysis, as they more accurately reflected the animals' response to being tickled. The Approach Behavior score (range 0-3) was related to positive vocalizations $(H(3)=$ $8.68, p=0.03$; Figure 3).

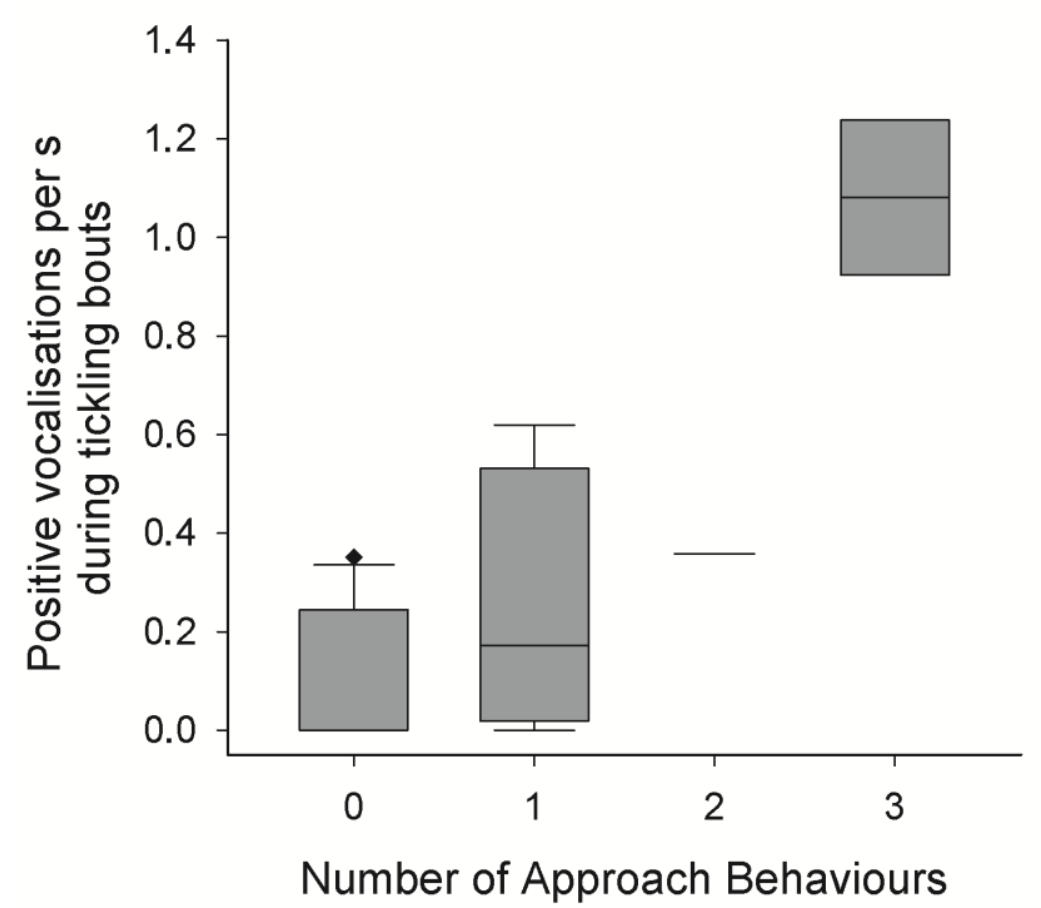

Figure 3. Association between positive vocalizations during tickling and approach behavior after tickling bouts in the Tickling test.

\section{Relationships Between Home Cage Social Play and Other Play Contexts}

The mean frequency of social play in the home cage during the first hour of the dark phase was $31.5 \pm 1.7$, of which $93.7 \%( \pm 2.2 \%)$ was observed in the first $30 \mathrm{~min}$ of darkness. Social play in the home cage was related to social play in the Play-In-Pairs test $r(18)=0.57, p=0.008$; Figure 4), yet positive vocalizations during tickling were unrelated to both social play in the home cage $\left(r_{s}=0.28, n . s.\right)$ and in the Play-In-Pairs test $\left(r_{s}=-0.07\right.$, n.s. $)$.

\section{Relationships Between Anxiety and Play Contexts}

All animals ate the sweetened cereal before the end of the Novelty-Induced Suppression-ofFeeding test. The number of fecal boli $(1.48 \pm 0.33$, range $0-4)$ and the latency to eat the cereal $(104.1 \pm$ $7.0 \mathrm{~s}$, range $45-150 \mathrm{~s})$ were correlated $\left(r_{s}=0.54, p=0.01\right)$. Production of fecal boli, being a more widely used proxy measure of anxiety (Archer, 1973; Ennaceur, Michalikova, \& Chazot, 2006), was used to investigate relationships with play contexts.

The number of fecal boli was unrelated to social play in the home cage $\left(r_{s}=0.12, n . s\right.$. $)$ and to social and solitary play in the Play-In-Pairs test $\left(r_{s}=0.15, n . s\right.$. , and $r_{s}=0.24$, n.s., respectively), yet tended to be negatively correlated with positive vocalizations during tickling $\left(r_{s}=-0.40, p=0.07\right)$. 


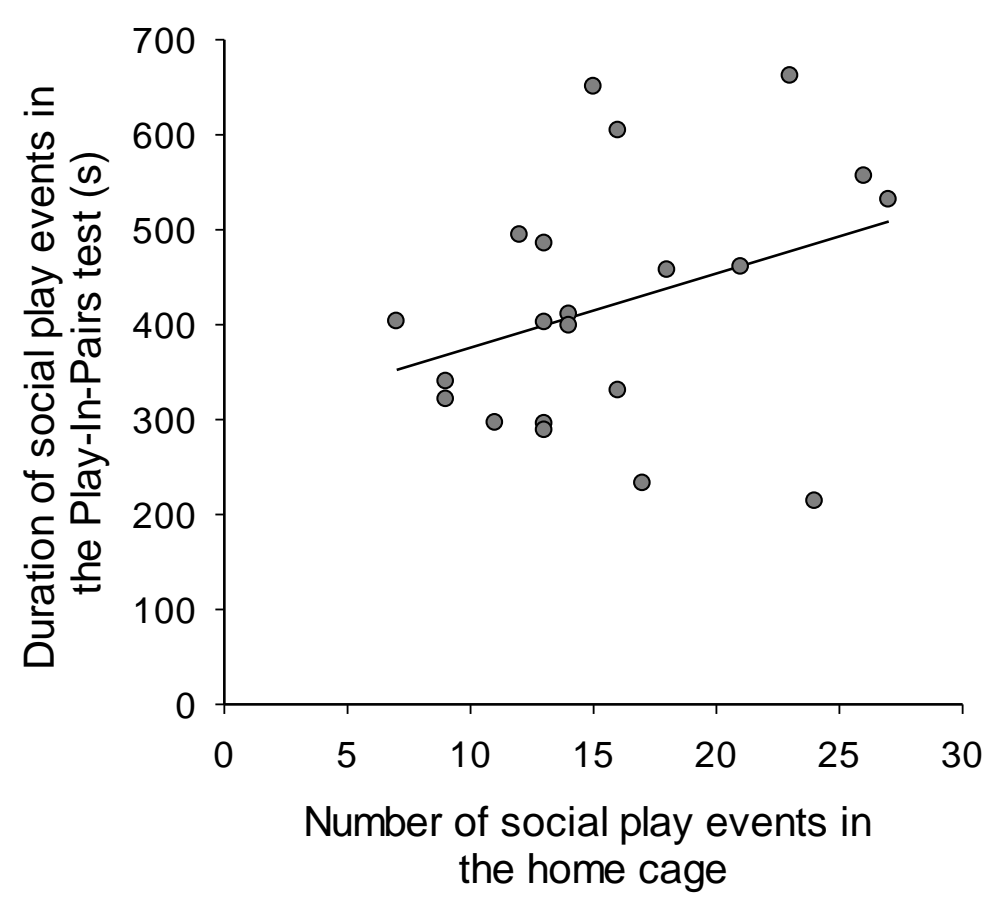

Figure 4. Correlation between the duration of social play in the Play-in-Pairs test and the frequency of social play events in the home cage.

\section{Discussion}

We found that play behavior in rats differed depending on the context; conspecific Rough-andTumble play seemed to be unrelated to vocalizations emitted during heterospecific play (manual tickling by an experimenter). Furthermore, we found that play types (social/solitary) were unrelated, and the sequence analysis of play structure revealed a difference between social and solitary play. In social play (Pinning/Being Pinned), repetitions of the same behaviors occurred more often than alternations between behaviors, while in solitary play (Scampering) no clear pattern of sequences was found.

\section{Conspecific and Heterospecific Play Contexts}

Individual playfulness in rats was consistent across two conspecific play contexts, namely play in the home cage in the first hour of the dark phase, and play of pairs of cage mates after a short isolation period. This result supports previous research suggesting that the Play-In-Pairs test can be a useful tool for a relatively quick assessment of playfulness (Niesink \& van Ree, 1989; Pellis \& McKenna, 1992; Vanderschuren, Stein et al., 1995). Measures of play in conspecific contexts, however, appeared to be unrelated to heterospecific play as measured by positive vocalizations induced by tickling, suggesting that the two play contexts may be independent. Although the recording at the individual level of positive vocalizations during conspecific play was not possible in this study, it would be plausible to expect that positive vocalizations at tickling, which have been associated to positive affect (Burgdorf \& Panksepp, 2001) and are highly emitted also in relation to play and sex (Burgdorf et al., 2008; Himmler, Kisko, Euston, Kolb, \& Pellis, 2014), would correlate with conspecific play behavior. Since preliminary observations indicated that initiations of play (i.e., dorsal contacts) and pinning behavior largely overlapped in this study, possibly due to the relatively small space available in the home cage, a comparison with previous studies in which dorsal contacts and pinning were analyzed separately is more difficult to make. While our result seem to confirm the finding of Panksepp and Burgdorf (1999) that 
conspecific pinning behavior is unrelated to positive vocalizations during tickling, we cannot definitely support or disprove their finding that conspecific play initiations are related to tickling vocalizations. However, our finding contrasts with Webber et al. (2012), who found some indications that different play behaviors were associated with animals selected for high and low levels of positive vocalizations during tickling (i.e., high responders performed more dorsal contacts while low responders performed more pinning behaviors on some testing days).

There are a few possible explanations for this result. On the one hand, play mates may vary in their intrinsic motivation to play (Pellis \& McKenna, 1992), and it has been suggested that if two individuals have similar motivation to play they are both more likely to perform high levels of play, while individuals with divergent motivation to play could result in the less motivated animal not necessarily participating in play, but rather avoiding it if given the possibility to do so (Douglas et al., 2004; Varlinskaya et al., 1999; Vanderschuren, 2010). In this way, some animals might have been involved in high levels of conspecific social play without this reflecting positive affect and therefore not relating to positive vocalizations at tickling. However, the effect of differing levels of motivation to play should be partially accounted for by looking at play levels with multiple partners in the Play-In-Pairs test. Also, Burgdorf et al. (2008) found that during conspecific play, the vast majority of vocalizations were positive, suggesting that any potential negative component of play may be minimal.

On the other hand, while conspecific play was unrelated to anxiety, as measured by the number of fecal boli produced in the Novelty-Induced Suppression-of-Feeding test, positive vocalizations during tickling tended to negatively correlate with anxiety. Mällo et al. (2007) did not find a difference in anxiety between high and low tickle responders, but frequency modulated $50 \mathrm{kHz}$ vocalizations were not distinguished from flat $50 \mathrm{KHz}$ ones, and the tickling procedure itself was found to have an anxiolytic effect in different test situations. However, similar to our findings, Burgdorf et al. (2009) found that rats selected for high tickle responding showed less anxiety in an open field test than low tickle responders. This suggests that positive vocalizations at tickling might reflect general lower levels of anxiety in the presence of the experimenter('s hand) and not necessarily levels of playfulness. In addition, since in previous studies rats were individually housed since weaning (Burgdorf et al., 2008; Panksepp \& Burgdorf, 1999), it is possible that social deprivation may have favored a correlation between heterospecific play vocalizations and conspecific play behavior by drastically increasing the motivation towards any social contact. Though tickling has been proposed to resemble conspecific Rough-andTumble play (Panksepp \& Burgdorf, 2000), it might not be perceived in a similar way by (socially housed) rats, and instead have divergent reward values at the individual level. Finally, since the Play-InPairs test and the Tickling test were performed 13 days apart from each other, it cannot be excluded that developmental changes in play behavior during this period could have masked a potential relationship between the two tests. While an overall decrease in pinning behavior would be expected between 41 and 54 days of age (Takahashi \& Lore, 1983; Thor \& Holloway, 1984), this would not necessarily affect individual consistency in playfulness. However, changes in ratio between the occurrence of play initiations (i.e., attacks to the nape) and playful defensive strategies (e.g., Pinning) have been shown to occur, though across a longer period of time (pre- vs. post- sexual maturity; Pellis \& Pellis, 1991). Such changes may partially explain previous age-dependant contrasting findings (Panksepp \& Burgdorf, 1999, see Section 1), therefore further research is needed before excluding any relationship between conspecific and heterospecific play.

\section{Social and Solitary Play Types}

Social play events (average between Pinning and Being Pinned frequencies) in the Play-In-Pairs test occurred more often than solitary play. This is most likely due to the fact that rats in the present study prioritized social play after a period of isolation, though it might be partially due to a general low frequency of Scampering behavior within a relatively small available space, similarly to low frequencies of running behavior compared to other locomotor-rotational behaviors after termination of social contact, as found by Pellis and Pellis (1983). Solitary play was unrelated to social play duration, suggesting that 
these play types are unrelated in rats, and supporting the theory that different types of play may have had independent evolutionary origins and trajectories (Burghardt, 2005). This is confirmed also by the fact that the proportions of each play behavior (on total play events) that one animal performed following solitary play of its cage mate were not found to be significantly different. This suggests that solitary play, which is not immediately directed at or induced by another individual, is not associated with the quantity of social play of the player or with the subsequent play behaviors of the play mate, at least for the play behaviors investigated in this study.

In addition, the Play-Role index positively correlated with Pinning duration but was unrelated to Being Pinned duration, indicating that when play roles were more different, higher overall levels of Pinning were performed. This result may relate to hierarchy formation, the onset of which already occurs during adolescence (Pellis \& McKenna, 1992), and might be driven by repetitions of Pinning by the animals which are to become dominant (Pellis \& McKenna, 1992; Pellis et al., 1997).

\section{Analysis of Play Structure}

Although the analysis of social play structure is limited to the measurement of pinning behavior in this study, the analysis of intra-individual dyadic sequences of behavior revealed that behavioral repetitions of the same social play behavior occurred more frequently than alternations, indicating that social play encounters were characterized by reiteration of either Pinning or Being Pinned behaviors, which occurred more frequently than inversions of play roles. This may support the fact that, although alternations of play roles are characteristic of social play in adolescent rats (Pellis et al., 1997), hierarchy formation was present and affected the play bouts already at the age of 41-43 days. In contrast, dyadic sequences involving solitary play did not follow a specific pattern.

\section{Novelty-Induced Suppression-of-Feeding and Tickling Tests}

In the Novelty-Induced Suppression-of-Feeding test, since the minimum latency for a rat to eat the sweetened cereal was 45 seconds, and up to four fecal boli were produced, we conclude that all animals experienced at least a moderate level of anxiety during testing.

The Tickling test revealed that positive frequency modulated $50 \mathrm{kHz}$ vocalizations emitted during tickling bouts highly correlated with those emitted in the remaining time of the tickling session. Panksepp and Burgdorf (2000) previously demonstrated that rats show a higher rate of $50 \mathrm{kHz}$ vocalizations in anticipation of tickling in a conditioned place preference test. Our results also suggest that alterations in affective state induced by tickling (or the anticipation of it) extended to the entire testing session. The number of Approach Behavior events which followed tickling positively correlated to positive vocalizations during tickling, confirming previous research showing that tickling is rewarding and is associated with positive affect (Burgdorf \& Panksepp, 2001). Although the animals emitted lower rates of positive vocalizations compared with previous studies (Burgdorf et al., 2008), possibly due to the short habituation to the tickling procedure, they did not produce fecal boli or emit negative vocalizations during testing, indicating that they did not find the procedure to be aversive. This is in contrast to Mällo et al. (2007) who reported emissions of negative vocalizations, which however were more frequent in female compared to male rats, and with Burgdorf et al. (2009) who reported that rats produced fecal boli during tickling.

In conclusion, play has been promoted as an indicator of positive affect in rats, however we propose that context, type, and differences in structure should be taken into account if play is to be used as a measure of positive welfare.

\section{Acknowledgements}

We would like to thank Prof. Louk Vandershuren for providing very useful advice on the preliminary results of this experiment, Jessica Lampe for helping with the editing of the manuscript, and two 
anonymous referees for their constructive comments. Our thanks also go to Zeljko Kragic for technical assistance with the experimental setup, and Myriam Siffert and Tina Sommerer for their assistance with animal husbandry. This study was funded by the Swiss National Science Foundation (Project $\mathrm{n}$. 31003A_144088).

\section{References}

Archer, J. (1973). Tests for emotionality in rats and mice: A review. Animal Behaviour, 21, 205-235.

Bekoff, M. (2001). The evolution of animal play, emotions, and social morality: On science, theology, spirituality, personhood, and love. Zygon, 36, 615-655.

Bodnoff, S. R., Suranyi-Cadotte, B., Quirion, R., \& Meaney, M. J. (1989). A comparison of the effects of diazepam versus several typical and atypical anti-depressant drugs in an animal model of anxiety. Psychopharmacology, 97, 277-279.

Boissy, A., Manteuffel, G., Jensen, M. B., Moe, R. O., Spruijt, B., Keeling, L. J., ...Aubert, A. (2007). Assessment of positive emotions in animals to improve their welfare: Stress and welfare in farm animals. Physiology \& Behavior, 92, 375-397.

Burgdorf, J., Kroes, R. A., Moskal, J. R., Pfaus, J. G., Brudzynski, S. M., \& Panksepp, J. (2008). Ultrasonic vocalizations of rats (Rattus norvegicus) during mating, play, and aggression: Behavioral concomitants, relationship to reward, and self-administration of playback. Journal of Comparative Psychology, 122, 357367.

Burgdorf, J., \& Panksepp, J. (2001). Tickling induces reward in adolescent rats. Physiology \& Behavior, 72, 167173.

Burgdorf, J., \& Panksepp, J. (2006). The neurobiology of positive emotions: The limbic brain: Structure and function. Neuroscience \& Biobehavioral Reviews, 30, 173-187.

Burgdorf, J., Panksepp, J., Brudzynski, S. M., Beinfeld, M. C., Cromwell, H. C., Kroes, R. A., \& Moskal, J. R. (2009). The effects of selective breeding for differential rates of $50-\mathrm{kHz}$ ultrasonic vocalizations on emotional behavior in rats. Developmental Psychobiology, 51, 34-46.

Burgdorf, J., Panksepp, J., \& Moskal, J. R. (2011). Frequency-modulated 50kHz ultrasonic vocalizations: A tool for uncovering the molecular substrates of positive affect: Pioneering research in affective neuroscience: Celebrating the work of Dr. Jaak Panksepp. Neuroscience \& Biobehavioral Reviews, 35, 1831-1836.

Burghardt, G. M. (2005). The genesis of animal play: Testing the limits. Cambridge, MA: MIT Press.

Douglas, L. A., Varlinskaya, E. I., \& Spear, L. P. (2004). Rewarding properties of social interactions in adolescent and adult male and female rats: Impact of social versus isolate housing of subjects and partners. Developmental Psychobiology, 45, 153-162.

Ennaceur, A., Michalikova, S., \& Chazot, P. (2006). Models of anxiety: Responses of rats to novelty in an open space and an enclosed space. Behavioural Brain Research, 171, 26-49.

Fagen, R. (1981). Animal play behavior. Oxford, UK: Oxford University Press.

Fraser, D., \& Duncan, I. J. H. (1998). 'Pleasures', 'pains' and animal welfare: Toward a natural history of affect. Animal Welfare, 7, 383-396.

Garvey, C. (1990). Play. Cambridge, MA: Harvard University Press.

Graham, K. L., \& Burghardt, G. M. (2010). Current perspectives on the biological study of play: Signs of progress. The Quarterly Review of Biology, 85, 393-418.

Held, S. D. E., \& Spinka, M. (2011). Animal play and animal welfare. Animal Behaviour, 81, 891-899.

Himmler, B. T., Kisko, T. M., Euston, D. R., Kolb, B., \& Pellis, S. M. (2014). Are 50-kHz calls used as play signals in the playful interactions of rats? I. Evidence from the timing and context of their use. Behavioural Processes, 106C, 60-66.

Hurst, J. L., Barnard, C. J., Hare, R., Wheeldon, E. B., \& West, C. D. (1996). Housing and welfare in laboratory rats: Time-budgeting and pathophysiology in single-sex groups. Animal Behaviour, 52, 335-360.

Klein, Z. A., Padow, V. A., \& Romeo, R. D. (2010). The effects of stress on play and home cage behaviors in adolescent male rats. Developmental Psychobiology, 52, 62-70.

Knutson, B., Burgdorf, J., \& Panksepp, J. (1998). Anticipation of play elicits high-frequency ultrasonic vocalizations in young rats. Journal of Comparative Psychology, 112, 65-73.

Knutson, B., Burgdorf, J., \& Panksepp, J. (2002). Ultrasonic vocalizations as indices of affective states in rats. Psychological Bulletin, 128, 961-977. 
Lawrence, A. (1987). Consumer demand theory and the assessment of animal welfare. Animal Behaviour, 35, 293295.

Mällo, T., Matrov, D., Herm, L., Kõiv, K., Eller, M., Rinken, A., \& Harro, J. (2007). Tickling-induced 50-kHz ultrasonic vocalization is individually stable and predicts behaviour in tests of anxiety and depression in rats. Behavioural Brain Research, 184, 57-71.

Niesink, R. J. M., \& van Ree, J. M. (1989). Involvement of opioid and dopaminergic systems in isolation-induced pinning and social grooming of young rats. Neuropharmacology, 28, 411-418.

Oliveira, A., Rossi, A., Silva, L., Lau, M., \& Barreto, R. (2010). Play behaviour in nonhuman animals and the animal welfare issue. Journal of Ethology, 28, 1-5.

Panksepp, J. (1981). The ontogeny of play in rats. Developmental Psychobiology, 14, 327-332.

Panksepp, J. (2005). Beyond a joke: From animal laughter to human joy? Science, 308, 62-63.

Panksepp, J., \& Beatty, W. W. (1980). Social deprivation and play in rats. Behavioral and Neural Biology, 30, 197206.

Panksepp, J., \& Burgdorf, J. (1999). Laughing rats? Playful tickling arouses high frequency ultrasonic chirping in young rodents. In S. Hameroff, C. Chalmers, \& A. Kazniak (Eds.), Toward a science of consciousness, Vol. 3 (pp. 231-244). Cambridge, MA: MIT Press.

Panksepp, J., \& Burgdorf, J. (2000). 50-kHz chirping (laughter?) in response to conditioned and unconditioned tickle-induced reward in rats: Effects of social housing and genetic variables. Behavioural Brain Research, $115,25-38$.

Pellis, S. M., Field, E. F., Smith, L. K., \& Pellis, V. C. (1997). Multiple differences in the play fighting of male and female rats. Implications for the causes and functions of play. Neuroscience \& Biobehavioral Reviews, 21, $105-120$.

Pellis, S. M., \& McKenna, M. M. (1992). Intrinsic and extrinsic influences on play fighting in rats: Effects of dominance, partner's playfulness, temperament and neonatal exposure to testosterone propionate. Behavioural Brain Research, 50, 135-145.

Pellis, S. M., \& Pellis, V. C. (1983). Locomotor-rotational movements in the ontogeny and play of the laboratory rat Rattus norvegicus. Developmental Psychobiology, 16, 269-86.

Pellis, S. M., \& Pellis, V. C. (1987). Play-fighting differs from serious fighting in both target of attack and tactics of fighting in the laboratory rat Rattus norvegicus. Aggressive Behavior, 13, 227-242.

Pellis, S. M., \& Pellis, V. C. (1991). Role reversal changes during the ontogeny of play fighting in male rats: Attack vs. defense. Aggressive Behavior, 17, 179-189.

Pellis, S. M., \& Pellis, V. C. (2007). Rough-and-tumble play and the development of the social brain. Current Directions in Psychological Science, 16, 95-98.

Spinka, M., Newberry, R. C., \& Bekoff, M. (2001). Mammalian play: Training for the unexpected. The Quarterly Review of Biology, 76, 141-168.

Takahashi, L. K., \& Lore, R. K. (1983). Play fighting and the development of agonistic behavior in male and female rats. Aggressive Behavior, 9, 217-227.

Thor, D. H., \& Holloway, W. R. (1984). Developmental analyses of social play behavior in juvenile rats. Bulletin of the Psychonomic Society, 22, 587-590.

Van der Poel, A. M., \& Miczek, K. (1991). Long ultrasonic calls in male rats following mating, defeat and aversive stimulation: Frequency modulation and bout structure. Behaviour, 119, 127-142.

Vanderschuren, L. J. M. J. (2010). How the brain makes play fun. American Journal of Play, 2, 315-337.

Vanderschuren, L. J. M. J., Niesink, R. J. M., Spruijt, B. M., \& van Ree, J. M. (1995). Influence of environmental factors on social play behavior of juvenile rats. Physiology \& Behavior, 58, 119-123.

Vanderschuren, L. J. M. J., Stein, E. A., Wiegant, V. M., \& van Ree, J. M. (1995). Social play alters regional brain opioid receptor binding in juvenile rats. Brain Research, 680, 148-156.

Varlinskaya, E. I., Spear, L. P., \& Spear, N. E. (1999). Social behavior and social motivation in adolescent rats: Role of housing conditions and partner's activity. Physiology \& Behavior, 67, 475-482.

Webber, E. S., Harmon, K. M., Beckwith, T. J., Peña, S., Burgdorf, J., Panksepp, J., \& Cromwell, H. C. (2012). Selective breeding for $50 \mathrm{kHz}$ ultrasonic vocalization emission produces alterations in the ontogeny and regulation of rough-and-tumble play. Behavioural Brain Research, 229, 138-44.

Wilson, S. C., \& Kleiman, D. G. (1974). Eliciting play: A comparative study. Integrative and Comparative Biology, $14,341-370$.

Wright, J. M., Gourdon, J. C., \& Clarke, P. B. S. (2010). Identification of multiple call categories within the rich repertoire of adult rat $50-\mathrm{kHz}$ ultrasonic vocalizations: Effects of amphetamine and social context. Psychopharmacology, 211, 1-13. 